\title{
Hydroxysafflor yellow A from safflower (Carthamus tinctorius L.) prevents cerebral ischemia-reperfusion injury in rats
}

\author{
Yunfeng WANG ${ }^{1}$, Yuanmin $\mathrm{DU}^{1}$, Jin $\mathrm{LI}^{1}$, Kang $\mathrm{XU}^{1 *}$
}

\begin{abstract}
In this study we extracted hydroxysafflor yellow A (HSYA) from safflower and investigate its preventive effect on cerebral ischemia-reperfusion injury (CIRI) in rats. HSYA with $89.78 \%$ purity was extracted from safflower. Ninety rats were divided into sham-operated, model, low-dose HSYA, high-dose HSYA and nimodipine groups. The CIRI model was established in latter three groups. After $30 \mathrm{~min}$ from ischemia beginning, the low-dose and high-dose HSYA groups were treated with 2 and $4 \mathrm{mg} / \mathrm{kg}$ HSYA via tail-vein injection, respectively. The nimodipine group was treated with $2 \mathrm{mg} / \mathrm{kg}$ nimodipine. Results showed that, at $24 \mathrm{~h}$ after ischemia, compared with model group, in high-dose HSYA and nimodipine groups the neurological symptom score was significantly decreased $(\mathrm{P}<0.05)$, the brain water content was significantly decreased $(\mathrm{P}<0.05)$, the brain infarction area percentage was significantly decreased $(\mathrm{P}<0.05)$, the serum IL- 6 and IL- $1 \beta$ levels were significantly decreased $(\mathrm{P}<0.05)$, and the brain tissue phosphorylated $\mathrm{p} 38$ mitogen-activated protein kinase ( $\mathrm{p}-\mathrm{p} 38 \mathrm{MAPK})$ and phosphorylated nuclear factor kappa $\mathrm{B}(\mathrm{p}-\mathrm{NF}-\mathrm{kB})$ p65 protein expression levels were significantly decreased $(\mathrm{P}<0.05)$. In conclusion, HSYA can inhibit the $\mathrm{p} 38 \mathrm{MAPK} / \mathrm{NF}-\mathrm{kB}$ pathway and reduce the inflammatory response, thus exerting the preventive effect on CIRI in rats.
\end{abstract}

Keywords: hydroxysafflor yellow A; cerebral ischemia-reperfusion injury; rats; p38 MAPK; NF-кB.

Practical Application: This study has provided a basis for obtaining hydroxysafflor yellow A from safflower and application of it to prevention of cerebral ischemia-reperfusion injury.

\section{Introduction}

Ischemic stroke has the high recurrence rate, disability rate and mortality rate, which seriously endangers the health and life of people (Skajaa et al., 2021). At present, the treatment principle for this disease is mainly to dissolve the thrombus and restore the blood supply. However, the reperfusion after cerebral ischemia can aggravate the pathological damage of ischemic brain tissues and worsen the cerebral injury, which is called the cerebral ischemia-reperfusion injury (CIRI) (Pan et al., 2007). Reducing CIRI has become an important link in the treatment of ischemic cerebrovascular diseases. The pathological mechanism of CIRI is complex. It involves the energy depletion, calcium overload, production of inflammatory mediators, production of free radicals, activation of apoptosis pathway, and so on (Cao \& Phillis, 1995; Zhao et al., 2013; Zhang et al., 2016; Liu \& Zhang, 2019). The inflammatory response plays a very important role in the physiological and pathological development of CIRI. Seeking drugs for preventing and treating CIRI through inhibiting the inflammatory response has become a research hotspot (Xian et al., 2019; Zheng et al., 2019). It is found that, p38 mitogen-activated protein kinase (p38 MAPK)/nuclear factor kappa B (NF- $\kappa$ B) is a classical inflammatory pathway, which can regulate the expression of a variety of inflammatory factors, thus regulating the inflammatory response (Li et al., 2012). Hydroxysafflor yellow A (HSYA) is the main active component of traditional Chinese medicine safflower (Carthamus tinctorius L.) (Bai et al., 2012).
Its molecular formula is $\mathrm{C}_{27} \mathrm{H}_{32} \mathrm{O}_{16}$, with molecular weight of 612.53. Pharmacological and clinical studies have shown that HSYA has the anti-inflammatory (Chen et al., 2008), antioxidant (Chen et al., 2016), platelet aggregation-inhibitory (Zang et al., 2002) and other effects. It is applied to treating of ischemic heart disease (Ji et al., 2009), preventing hypoxia injury (Ye \& Gao, 2008) and protecting against chronic liver fibrosis (Zhang et al., 2011). In view of this, this study intended to extract HSYA from safflower and investigate the preventive effect of HSYA on CIRI in rats and the related mechanisms.

\section{Materials and methods}

\subsection{Extraction of HSYA}

Safflower powder (500 g) was added into microwave digestion tank, followed by adding $10 \mathrm{~L}$ of $50 \%$ ethanol-water solution. After vortex shaking for $1 \mathrm{~min}$, the microwave-assisted extraction was performed for $30 \mathrm{~min}$. After cooling to room temperature, the mixture was filtered. The filtrate was concentrated by vacuum. The crude extract was obtained, and was dissolved in water. After standing at $4{ }^{\circ} \mathrm{C}$ over night, the mixture was filtered. The filtrate was loaded to $\mathrm{X}-5$ macroporous resin column. The concentration of HSYA in load sample was adjusted to $1.0 \mathrm{mg} / \mathrm{mL}$, and the load flow rate was $10 \mathrm{BV} / \mathrm{h}$. Then, $70 \%$ ethanol-water solution was used for elution, and the elution flow rate was $5 \mathrm{BV} / \mathrm{h}$. The effluent 
was collected, and concentrated by vacuum. The concentrated product was dissolved in water, followed by load on silica gel column. The elution was performed using ethyl acetate-95\% ethanol (3:1) solution. The effluent was collected, followed by vacuum concentration and freeze drying. Finally, the refined HSYA product was obtained. The high performance liquid chromatography showed that the purity of HSYA was $89.78 \%$.

\subsection{Establishment of CIRI model.}

Ninety Sprague Dawley rats (280-300 g) were randomly divided into sham-operated, model, low-dose HSYA, high-dose HSYA and nimodipine groups, with 18 rats in each group. The CIRI model was established in latter four groups using the right middle cerebral artery embolization (MCAO) method. The rats were anesthetized with isoflurane. A median incision was made in the neck. The common carotid artery, external carotid artery and internal carotid artery were isolated. The internal carotid artery was clamped using the arterial clamper. An incision was made at the bifurcation of external carotid artery and internal carotid artery. A nylon thread was inserted into the internal carotid artery for about $22 \mathrm{~mm}$ distance until there was a slight sense of resistance. After $2 \mathrm{~h}$ of occlusion for ischemia, the nylon thread was removed for reperfusion. In sham-operated group, the surgical operations were the same as those in other groups, excepting insertion of nylon thread into internal carotid artery. The room temperature was kept at $23-25^{\circ} \mathrm{C}$ during the surgery.

\subsection{Treatment}

After $30 \mathrm{~min}$ from the ischemia beginning, the rats in low-dose HSYA group and high-dose HSYA group were treated with 2 and $4 \mathrm{mg} / \mathrm{kg}$ HSYA via tail-vein injection, respectively. The rats in nimodipine group were treated with $2 \mathrm{mg} / \mathrm{kg}$ nimodipine via tail-vein injection. The rats in sham-operated group and model group were treated with the same volume of normal saline via tail-vein injection.

\subsection{Neurological symptom scoring}

After $3 \mathrm{~h}, 6 \mathrm{~h} 12$ and $24 \mathrm{~h}$ from ischemia, the neurological symptoms of rats were evaluated according to the scoring system as follows: 0 point: no symptom of neurological deficit, normal activity; 1 point: the rats could not fully extend the forepaws on hemiplegic side; 2 points: the rats turned around to the hemiplegic side; 3 points: the rats tilted to the hemiplegic side; 4 points: the rats could not spontaneously walk, with loss of consciousness; 5 points: the rats died.

\subsection{Determination of serum inflammatory factors}

After the last neurological symptom scoring, the rats were anaesthetized with $10 \%$ chloral hydrate. The blood was collected from the heart in supine position. The blood samples were centrifuged at $1500 \mathrm{r} / \mathrm{min}$ for $20 \mathrm{~min}$, the serum was taken and stored at $-20^{\circ} \mathrm{C}$ for testing. The serum interleukin 6 (IL-6) interleukin $1 \beta$ (IL- $\beta$ ) levels were determined using enzyme-linked immunosorbent assay. The operations were according to the instructions of kits

\subsection{Measurement of brain water content}

Six rats in each group were randomly taken. The rats were sacrificed, and the brain was immediately taken. The brain was rinsed with normal saline. The liquid on the surface was sucked dry using filter paper. The brain was weighed to obtain the wet mass. Then, the brain was dried in an oven at $100^{\circ} \mathrm{C}$ to constant weight to obtain the dry mass. The brain water content was calculated as follows: brain water content $(\%)=[$ (wet mass - dry mass) / wet mass] $\times 100 \%$.

\subsection{Determination of brain infarction area}

Six rats in each group were randomly taken. The rats were sacrificed, and the brain was immediately taken. The brain tissue slices with $2 \mathrm{~mm}$ thickness were prepared. The slices were stained in $1 \%$ 2,3,5-triphenyltetrazolium chloride solution at $37^{\circ} \mathrm{C}$ for $20 \mathrm{~min}$, followed by fixation with $10 \%$ paraformaldehyde. The normal brain tissue area presented rose red, and the infarction area presented white. The sections were photographed and analyzed using the ImageJ software. The percentage of brain infarction area was calculated.

\subsection{Western blotting}

The remaining six rats in each group were sacrificed, and the brain was immediately taken. The brain tissues were homogenized with RIPA lysate. After centrifugation, the supernatant was extracted and the protein content was determined by bicinchonininc acid method. A $20 \mu \mathrm{g}$ of crude protein sample was loaded on sodium dodecyl sulfonate-polyacrylamide gel electrophoresis. The separated proteins were transferred to the polyvinylidene fluoride membranes. After blocking using $1 \%$ bovine serum albumin, the membranes were incubated with primary antibody (anti-p-p38 MAPK, 1: 1000; anti-p-NF- $\kappa B$ p65, 1: 2000; anti-glyceraldehyde-3-phosphate dehydrogenase (GAPDH), 1:5000) at $4^{\circ} \mathrm{C}$ overnight, respectively. After rinsing with Tris buffered saline Tween (TBST) for three times, the membranes were incubated with the second antibody horseradish peroxidase-labeled anti-IgG $(1: 2000)$ at $37^{\circ} \mathrm{C}$ for $1.5 \mathrm{~h}$. After rinsing with TBST for three times, the membranes were visualized using the enhanced chemiluminescence reagent. The absorbance of strips was measured by Image J software. Using GAPDH as internal reference, the expression level of the target protein is expressed according to the absorbance ratio of the target protein strip to the GAPDH strip.

\subsection{Statistical analysis}

The analysis was performed using SPSS 20.0 statistical software. All data were represented as mean \pm standard deviation. The data were analyzed using one-way analysis of variance followed by Bonferroni test to determine the difference among groups. Significant differences were accepted for $\mathrm{P}<0.05$.

\section{Results}

\subsection{Comparison of neurological symptom score among five groups}

Table 1 showed that, at $3 \mathrm{~h}, 6 \mathrm{~h} 12$ and $24 \mathrm{~h}$ after ischemia, the neurological symptom score in model, low-dose HSYA, high-dose HSYA and nimodipine groups was obviously higher 
Table 1. Comparison of neurological symptom score among five groups $(n=18)$.

\begin{tabular}{|c|c|c|c|c|}
\hline \multirow{2}{*}{ Group } & \multicolumn{4}{|c|}{ Neurological symptom score (points) } \\
\hline & $3 \mathrm{~h}$ & $6 \mathrm{~h}$ & $12 \mathrm{~h}$ & $24 \mathrm{~h}$ \\
\hline Sham-operated & $0.00 \pm 0.00$ & $0.00 \pm 0.00$ & $0.00 \pm 0.00$ & $0.00 \pm 0.00$ \\
\hline Model & $2.83 \pm 0.23^{\#}$ & $3.51 \pm 0.48^{*}$ & $3.97 \pm 0.63^{*}$ & $4.32 \pm 0.81^{\#}$ \\
\hline Low-dose HSYA & $2.80 \pm 0.47^{\sharp}$ & $3.12 \pm 0.31^{\# \$}$ & $3.56 \pm 0.52^{*}$ & $3.47 \pm 0.77^{\sharp \$}$ \\
\hline High-dose HSYA & $2.59 \pm 0.42^{\# \$}$ & $2.75 \pm 0.69^{\# \$ \%}$ & $3.27 \pm 0.49^{\# \$}$ & $2.82 \pm 0.59^{\# 5 \%}$ \\
\hline Nimodipine & $2.53 \pm 0.36^{\# 5 \%}$ & $2.61 \pm 0.47^{\# \$ \%}$ & $2.88 \pm 0.58^{\# \% \wedge}$ & $2.62 \pm 0.43^{\# 5 \%}$ \\
\hline
\end{tabular}

Data were represented as mean \pm standard deviation. ${ }^{*} \mathrm{P}<0.05$ compared with sham-operated group; ${ }^{\$} \mathrm{P}<0.05$ compared with model group; ${ }^{*} \mathrm{P}<0.05$ compared with low-dose HSYA group; ${ }^{\wedge} \mathrm{P}<0.05$ compared with high-dose HSYA group. HSYA, hydroxysafflor yellow A.

than that in sham-operated group, respectively $(\mathrm{P}<0.05)$. Compared with model group, the neurological symptom score in high-dose HSYA and nimodipine groups at $3 \mathrm{~h}$ and $12 \mathrm{~h}$ and in low-dose HSYA, high-dose HSYA and nimodipine groups at $6 \mathrm{~h}$ and $24 \mathrm{~h}$ were significantly decreased, respectively $(\mathrm{P}<0.05)$.

\subsection{Comparison of brain water content among five groups}

At $24 \mathrm{~h}$ after ischemia, the brain water content in sham-operated, model, low-dose HSYA, high-dose HSYA and nimodipine groups was $(55.18 \pm 7.69) \%,(85.20 \pm 7.80) \%$, $(82.20 \pm 9.13) \%$, $(70.12 \pm 9.59) \%$ and $(68.90 \pm 5.29) \%$, respectively. Compared with sham-operated group, the brain water content in model, low-dose HSYA, high-dose HSYA and nimodipine groups was obviously increased, respectively $(\mathrm{P}<0.05)$. Compared with model group, the brain water content in high-dose HSYA and nimodipine groups was significantly decreased, respectively $(\mathrm{P}<0.05)$ (Figure 1).

\subsection{Comparison of percentage of brain infarction area among five groups}

At $24 \mathrm{~h}$ after ischemia, percentage of brain infarction area in sham-operated, model, low-dose HSYA, high-dose HSYA and nimodipine groups was $(0.00 \pm 0.00) \%,(46.34 \pm 5.87) \%$, $(40.76 \pm 7.32) \%,(38.18 \pm 5.76) \%$ and $(33.39 \pm 4.45) \%$, respectively. There was no brain infarction area in sham-operated group, with obvious brain infarction area in model, low-dose HSYA, high-dose HSYA and nimodipine groups. Compared with model group, the percentage of brain infarction area in high-dose HSYA and nimodipine groups was significantly decreased, respectively $(\mathrm{P}<0.05)$ (Figure 2).

\subsection{Comparison of serum IL-6 and IL-1 $\beta$ levels among five groups}

At $24 \mathrm{~h}$ after ischemia, compared with sham-operated group, the serum IL-6 and IL-1 $\beta$ levels in model, low-dose HSYA, high-dose HSYA and nimodipine groups was obviously increased, respectively $(\mathrm{P}<0.05)$. Compared with model group, the serum IL- 6 and IL- $1 \beta$ levels in low-dose HSYA, high-dose HSYA and nimodipine groups were significantly decreased, respectively $(\mathrm{P}<0.05)$. In addition, the serum IL- $1 \beta$ level in high-dose HSYA group was significantly lower than that in nimodipine group $(\mathrm{P}<0.05)$ (Table 2$)$.

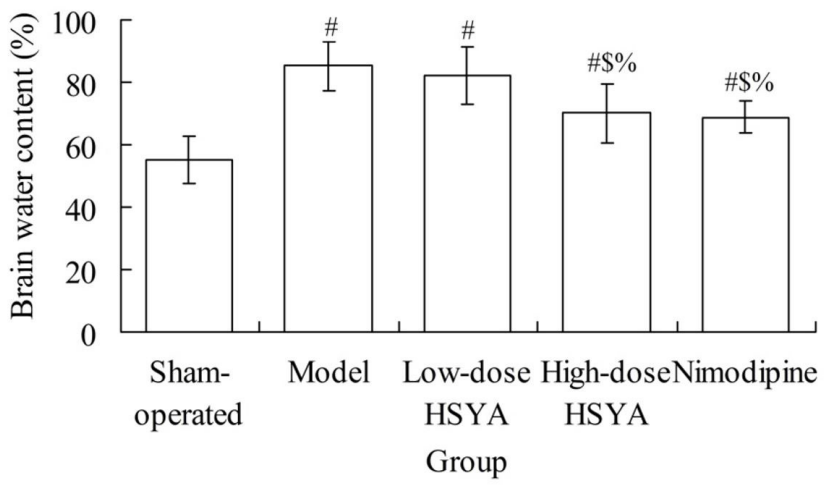

Figure 1. Comparison of brain water content among five groups $(\mathrm{n}=6)$. Data were represented as mean \pm standard deviation. ${ }^{*} \mathrm{P}<0.05$ compared with sham-operated group; ${ }^{\$} \mathrm{P}<0.05$ compared with model group; ${ }^{\%} \mathrm{P}<0.05$ compared with low-dose HSYA group. HSYA, hydroxysafflor yellow A.

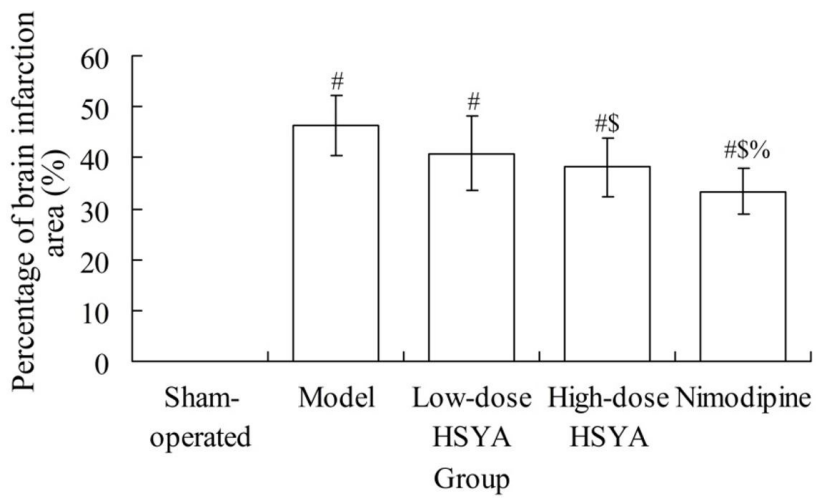

Figure 2. Comparison of percentage of brain infarction area among five groups $(n=6)$. Data were represented as mean \pm standard deviation. ${ }^{\#} \mathrm{P}<0.05$ compared with sham-operated group; ${ }^{\text {P }}<0.05$ compared with model group; ${ }^{\%} \mathrm{P}<0.05$ compared with low-dose HSYA group. HSYA, hydroxysafflor yellow A.

\subsection{Comparison of brain tissue p-p38 MAPK and $p-N F-\kappa B$ p65 protein expression levels among five groups}

Table 3 showed that, at $24 \mathrm{~h}$ after ischemia, the brain tissue p-p38 MAPK and p-NF- $\kappa$ B p65 protein expression levels in model, low-dose HSYA, high-dose HSYA and nimodipine groups were obviously higher than those in sham-operated 
Table 2. Comparison of serum IL-6 and IL- $1 \beta$ levels among five groups $(\mathrm{n}=18)$.

\begin{tabular}{clc}
\hline Group & IL-6 $(\mathrm{pg} / \mathrm{mL})$ & IL-1 $\beta(\mathrm{pg} / \mathrm{mL})$ \\
\hline Sham-operated & $25.38 \pm 3.56$ & $7.34 \pm 1.52$ \\
Model & $55.76 \pm 7.27^{\#}$ & $16.10 \pm 2.39^{\sharp}$ \\
Low-dose HSYA & $50.29 \pm 8.20^{\# \$}$ & $13.67 \pm 1.80^{\sharp \$}$ \\
High-dose HSYA & $36.15 \pm 4.19^{* \$ \%}$ & $10.09 \pm 0.78^{\# \%}$ \\
Nimodipine & $33.26 \pm 5.84^{\# \$ \%}$ & $12.86 \pm 1.14^{\# \wedge}$ \\
\hline
\end{tabular}

Data were represented as mean \pm standard deviation. ${ }^{*} \mathrm{P}<0.05$ compared with sham-operated group; ${ }^{s} \mathrm{P}<0.05$ compared with model group; ${ }^{\%} \mathrm{P}<0.05$ compared with low-dose HSYA group; ${ }^{\wedge} \mathrm{P}<0.05$ compared with high-dose HSYA group. HSYA, hydroxysafflor yellow A; IL-6, interleukin 6; IL- $\beta$, interleukin $1 \beta$.

Table 3. Comparison of brain tissue p-p38 MAPK and p-NF- $\mathrm{BB}$ p65 protein expression levels among five groups $(n=6)$.

\begin{tabular}{|c|c|c|}
\hline Group & p-p38 MAPK/GAPDH & p-NF- $\kappa$ B p65/GAPDH \\
\hline Sham-operated & $0.34 \pm 0.06$ & $0.45 \pm 0.07$ \\
\hline Model & $0.88 \pm 0.11^{\#}$ & $1.45 \pm 0.12^{\#}$ \\
\hline $\begin{array}{l}\text { Low-dose } \\
\text { HSYA }\end{array}$ & $0.74 \pm 0.09^{\# \$}$ & $1.23 \pm 0.17^{\# \$}$ \\
\hline $\begin{array}{c}\text { High-dose } \\
\text { HSYA }\end{array}$ & $0.52 \pm 0.05^{\# \% \%}$ & $0.82 \pm 0.07^{\# \$ \%}$ \\
\hline Nimodipine & $0.46 \pm 0.06^{\# \$ \%}$ & $0.66 \pm 0.05^{\# \$ \%^{\wedge}}$ \\
\hline
\end{tabular}

Data were represented as mean \pm standard deviation. ${ }^{*} \mathrm{P}<0.05$ compared with shamoperated group; ${ }^{\$} \mathrm{P}<0.05$ compared with model group; ${ }^{\%} \mathrm{P}<0.05$ compared with low-dose HSYA group; ${ }^{\wedge} \mathrm{P}<0.05$ compared with high-dose HSYA group. HSYA, hydroxysafflor yellow A; p-p38 MAPK, phosphorylated p38 mitogen-activated protein kinase; p-NF- $\kappa B$ p65, phosphorylated nuclear factor kappa B p65; GAPDH, glyceraldehyde-3-phosphate dehydrogenase.

group, respectively $(\mathrm{P}<0.05)$. Compared with model group, the p-p38 MAPK and p-NF- $\kappa B$ p65 levels in low-dose HSYA, high-dose HSYA and nimodipine groups were significantly decreased, respectively $(\mathrm{P}<0.05)$. In addition, the brain tissue $\mathrm{p}-\mathrm{NF}-\kappa \mathrm{B}$ p65 level in nimodipine group was significantly lower than that in high-dose HSYA group $(\mathrm{P}<0.05)$.

\section{Discussion}

In this study, HSYA was successfully extracted from safflower. The purity of HSYA product was $89.78 \%$. This shows that the HSYA extraction technology in this study is feasible. CIRI is the secondary damage to brain tissue caused by cascade reaction due to blood flow reperfusion. It often leads to the neurological deficit, cerebral infarction and brain edema, and can cause the hemiplegia, aphasia and even death in severe cases (Wang et al., 2017a). This study investigated the preventive effect of HSYA on CIRI in rats. Results showed that, compared with model group, in HSYA groups the neurological symptom score, brain water content and percentage of brain infarction area were obviously decreased. It is suggested that HSYA can alleviate the CIRI in rats, thus exerting certain neuroprotective effect.

Inflammatory response plays an important role in CIRI (Zhong et al., 2019). Many inflammatory cells and mediators are involved in the inflammatory response. Inhibition of inflammatory response and reduction of brain tissue damage is one of the important means for treatment of stroke (Duris et al., 2018).
Interleukin is produced by a variety of cells and plays an important role in inflammatory response and humoral immunity. IL- 6 can induce the leukocyte adhesion, and activate the complement, thus blocking the microcirculation and damaging the tissue cells (Lokau et al., 2017). IL-1 $\beta$ is an important medium to trigger the immune and inflammatory responses. Study has shown that IL- $1 \beta$ is in a biphasic release mode in the CIRI model, and its level is obviously increased in CIRI (Yang et al., 2016). In the present study, at $24 \mathrm{~h}$ after ischemia, compared with shamoperated group, the serum IL- 6 and IL- $1 \beta$ levels in other groups was obviously increased. This confirms that the inflammatory response is involved in CIRI. Compared with model group, the serum IL-6 and IL-1 $\beta$ levels HSYA groups were significantly decreased. This suggests that, HSYA can reduce the inflammatory response, thus alleviating the CIRI in rats.

NF- $\kappa B$ is an important transcription activator, and its regulatory genes can encode the cytokines, immune regulatory molecules, etc.. It participates in the inflammatory response ( $\mathrm{Li}$ et al., 2008). During cerebral ischemia, NF- $\kappa B$ is phosphorylated by inflammatory factors, cytokines, calcium overload and other factors. The p-NF- $\mathrm{kB}$ can induce the expression of cytokines, adhesion molecules, and inflammatory enzymes, forming a vicious circle of inflammatory response, which leads to brain tissue edema and nerve cell damage (Zhang et al., 2005; Ridder \& Schwaninger, 2009). p38 MAPK, as a member of MAPK family, plays an important role in inflammatory response and its regulation ( $\mathrm{Ki}$ et al., 2013). After stimulation, p38 MAPK is phosphorylated to form a specific substrate p-p38 MAPK. $\mathrm{p}-\mathrm{p} 38 \mathrm{MAPK}$ is the upstream signal molecule of NF- $\mathrm{KB}$. It can promote the phosphorylation of NF- $\mathrm{kB}$ to form $\mathrm{p}-\mathrm{NF}-\mathrm{\kappa B}$ p65, thus regulating the transcription of inflammatory genes (Wang et al., 2017b). Results of our study showed that, the brain tissue p-p38 MAPK and p-NF- $\mathrm{kB}$ p 65 protein expression levels in CIRI rats were obviously higher than sham-operated rats. This indicates that, the activation of $\mathrm{p} 38$ MAPK/NF- $\kappa \mathrm{B}$ signal pathway is involved in the CIRI of rats. Compared with model group, the p-p38 MAPK and p-NF- $\kappa$ B p65 levels HSYA groups were significantly decreased. This indicates that, the HSYA can inhibit the p38 MAPK/NF- $\kappa B$ pathway to reduce the inflammatory response in CIRI rats.

\section{Conclusion}

In conclusion, HSYA product with $89.78 \%$ purity is successfully extracted from safflower. HSYA can inhibit the p38 MAPK/NF- $\kappa B$ pathway and reduce the inflammatory response, thus exerting the preventive effect on CIRI in rats. This study may provide a reference for further clarifying the action mechanism of HSYA in prevention of CIRI. This study still has some limitations. Firstly, the sample size of this study is relatively small. Secondly, other mechanisms related to the prevention of HSYA on CIRI have not been investigated. These issues should be solved in further studies.

\section{References}

Bai, Y., Lu, P., Han, C., Yu, C., Chen, M., He, F., Yi, D., \& Wu, L. (2012). Hydroxysafflor yellow A (HSYA) from flowers of Carthamus tinctorius L. and its vasodilatation effects on pulmonary artery. Molecules, 
17(12), 14918-14927. http://dx.doi.org/10.3390/molecules171214918. PMid:23519263.

Cao, X., \& Phillis, J. W. (1995). The free radical scavenger, alphalipoic acid, protects against cerebral ischemia-reperfusion injury in gerbils. Free Radical Research, 23(4), 365-370. http://dx.doi. org/10.3109/10715769509065257. PMid:7493042.

Chen, M., Wang, M., Yang, Q., Wang, M., Wang, Z., Zhu, Y., Zhang, Y., Wang, C., Jia, Y., Li, Y., \& Wen, A. (2016). Antioxidant effects of hydroxysafflor yellow A and acetyl-11-keto- $\beta$-boswellic acid in combination on isoproterenol-induced myocardial injury in rats. International Journal of Molecular Medicine, 37(6), 1501-1510. http:// dx.doi.org/10.3892/ijmm.2016.2571. PMid:27121241.

Chen, T. T., Du, Y. J., Liu, X. L., \& Zhu, H. B. (2008). Inhibitory action of hydroxysafflor yellow A on inflammatory signal transduction pathway related factors in rats with cerebral cortex ischemia. Yao Xиe Xue Bao, 43(6), 570-575. PMid:18822957.

Duris, K., Splichal, Z., \& Jurajda, M. (2018). The role of inflammatory response in stroke associated programmed cell Death. Current Neuropharmacology, 16(9), 1365-1374. http://dx.doi.org/10.2174/ 1570159X16666180222155833. PMid:29473512.

Lokau, J., Wandel, M., \& Garbers, C. (2017). Enhancing Interleukin-6 and Interleukin-11 receptor cleavage. The International Journal of Biochemistry \& Cell Biology, 85, 6-14. http://dx.doi.org/10.1016/j. biocel.2017.01.014. PMid:28143719.

Ji, D. B., Zhang, L. Y., Li, C. L., Ye, J., \& Zhu, H. B. (2009). Effect of Hydroxysafflor yellow A on human umbilical vein endothelial cells under hypoxia. Vascular Pharmacology, 50(3-4), 137-145. http:// dx.doi.org/10.1016/j.vph.2008.11.009. PMid:19084079.

Ki, Y. W., Park, J. H., Lee, J. E., Shin, I. C., \& Koh, H. C. (2013). JNK and p38 MAPK regulate oxidative stress and the inflammatory response in chlorpyrifos-induced apoptosis. Toxicology Letters, 218(3), 235245. http://dx.doi.org/10.1016/j.toxlet.2013.02.003. PMid:23416140.

Li, W., Khor, T. O., Xu, C., Shen, G., Jeong, W. S., Yu, S., \& Kong, A. N. (2008). Activation of Nrf2-antioxidant signaling attenuates NFkB-inflammatory response and elicits apoptosis. Biochemical Pharmacology, 76(11), 1485-1489. http://dx.doi.org/10.1016/j. bcp.2008.07.017. PMid:18694732.

Li, X., Zheng, Z., Li, X., \& Ma, X. (2012). Unfractionated heparin inhibits lipopolysaccharide-induced inflammatory response through blocking p38 MAPK and NF- $\kappa B$ activation on endothelial cell. Cytokine, 60(1), 114-121. http://dx.doi.org/10.1016/j.cyto.2012.06.008. PMid:22763042.

Liu, Q., \& Zhang, Y. (2019). PRDX1 enhances cerebral ischemia-reperfusion injury through activation of TLR4-regulated inflammation and apoptosis. Biochemical and Biophysical Research Communications, 519(3), 453-461. http://dx.doi.org/10.1016/j.bbrc.2019.08.077. PMid:31526567.

Pan, J., Konstas, A. A., Bateman, B., Ortolano, G. A., \& Pile-Spellman, J. (2007). Reperfusion injury following cerebral ischemia: pathophysiology, MR imaging, and potential therapies. Neuroradiology, 49(2), 93-102. http://dx.doi.org/10.1007/s00234-006-0183-z. PMid:17177065.

Ridder, D. A., \& Schwaninger, M. (2009). NF-kappaB signaling in cerebral ischemia. Neuroscience, 158(3), 995-1006. http://dx.doi. org/10.1016/j.neuroscience.2008.07.007. PMid:18675321.

Skajaa, N., Adelborg, K., Horváth-Puhó, E., Rothman, K. J., Henderson, V. W., Casper Thygesen, L., \& Toft Sørensen, H. (2021). Nationwide trends in incidence and mortality of stroke among younger and older adults in Denmark. Neurology, 96(13), e1711-e1723. http://dx.doi. org/10.1212/WNL.0000000000011636. PMid:33568547.
Wang, X., An, F., Wang, S., An, Z., \&Wang, S. (2017a). Orientin attenuates cerebral ischemia: reperfusion injury in rat model through the AQP-4 and TLR4/NF- $\mathrm{kB} / \mathrm{TNF}-\alpha$ signaling pathway.Journal of Stroke and Cerebrovascular Diseases, 26(10), 2199-2214. http://dx.doi. org/10.1016/j.jstrokecerebrovasdis.2017.05.002. PMid:28645524.

Wang, Y. Y., Ryu, A. R., Jin, S., Jeon, Y. M., \&Lee, M. Y. (2017b). Chlorin e6-Mediated Photodynamic therapy suppresses $P$. acnes-induced inflammatory response via NFKB and MAPKs signaling pathway. PLoS One, 12(1), e0170599. http://dx.doi.org/10.1371/journal. pone.0170599. PMid:28118375.

Xian, W., Li, T., Li, L., Hu, L., \& Cao, J. (2019). Maresin 1 attenuates the inflammatory response and mitochondrial damage in mice with cerebral ischemia/reperfusion in a SIRT1-dependent manner. Brain Research, 1711, 83-90. http://dx.doi.org/10.1016/j.brainres.2019.01.013. PMid:30639123.

Yang, Z., Weian, C., Susu, H., \& Hanmin, W. (2016). Protective effects of mangiferin on cerebral ischemia-reperfusion injury and its mechanisms. European Journal of Pharmacology, 771, 145-151. http://dx.doi.org/10.1016/j.ejphar.2015.12.003. PMid:26656757.

Ye, S. Y., \& Gao, W. Y. (2008). Hydroxysafflor yellow A protects neuron against hypoxia injury and suppresses inflammatory responses following focal ischemia reperfusion in rats. Archives of Pharmacal Research, 31(8), 1010-1015. http://dx.doi.org/10.1007/s12272-0011261-y. PMid:18787790.

Zang, B. X., Jin, M., Si, N., Zhang, Y., Wu, W., \& Piao, Y. Z. (2002). Antagonistic effect of hydroxysafflor yellow A on the platelet activating factor receptor. Yao Xue Xue Bao, 37(9), 696-699. PMid:12567893.

Zhang, W., Potrovita, I., Tarabin, V., Herrmann, O., Beer, V., Weih, F., Schneider, A., \& Schwaninger, M. (2005). Neuronal activation of NF-kappaB contributes to cell death in cerebral ischemia. Journal of Cerebral Blood Flow and Metabolism, 25(1), 30-40. http://dx.doi. org/10.1038/sj.jcbfm.9600004. PMid:15678110.

Zhang, Y., Guo, J., Dong, H., Zhao, X., Zhou, L., Li, X., Liu, J., \& Niu, Y. (2011). Hydroxysafflor yellow A protects against chronic carbon tetrachloride-induced liver fibrosis. European Journal of Pharmacology, 660(2-3), 438-444. http://dx.doi.org/10.1016/j.ejphar.2011.04.015. PMid:21536026.

Zhang, Y., Cao, R. Y., Jia, X., Li, Q., Qiao, L., Yan, G., \& Yang, J. (2016). Treadmill exercise promotes neuroprotection against cerebral ischemia-reperfusion injury via downregulation of pro-inflammatory mediators. Neuropsychiatric Disease and Treatment, 12, 3161-3173. http://dx.doi.org/10.2147/NDT.S121779. PMid:28003752.

Zhao, Q., Wang, S., Li, Y., Wang, P., Li, S., Guo, Y., \& Yao, R. (2013). The role of the mitochondrial calcium uniporter in cerebral ischemia/ reperfusion injury in rats involves regulation of mitochondrial energy metabolism. Molecular Medicine Reports, 7(4), 1073-1080. http:// dx.doi.org/10.3892/mmr.2013.1321. PMid:23426506.

Zheng, T., Jiang, H., Jin, R., Zhao, Y., Bai, Y., Xu, H., \& Chen, Y. (2019). Ginsenoside Rg1 attenuates protein aggregation and inflammatory response following cerebral ischemia and reperfusion injury. European Journal of Pharmacology, 853, 65-73. http://dx.doi.org/10.1016/j. ejphar.2019.02.018. PMid:30794781.

Zhong, Y., Yu, C., \& Qin, W. (2019). LncRNA SNHG14 promotes inflammatory response induced by cerebral ischemia/reperfusion injury through regulating miR-136-5p /ROCK1. Cancer Gene Therapy, 26(7-8), 234-247. http://dx.doi.org/10.1038/s41417-0180067-5. PMid:30546117. 